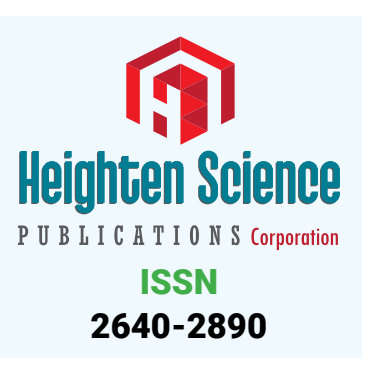

*Address for Correspondence: Sarmad Muhammad Soomar, Senior Research Assistant, Department of Psychiatry, Aga Khan University, Karachi, Pakistan, Tel: +92 33223835 09;

Email: soomarsarmad@gmail.com

Submitted: 19 March 2019

Approved: 01 April 2019

Published: 02 April 2019

Copyright: @ 2019 Soomar MS, et al. This is an open access article distributed under the Creative Commons Attribution License, which permits unrestricted use, distribution, and reproduction in any medium, provided the original work is properly cited

Check for updates
Opinion

\section{Managing epileptic women in pregnancy}

\author{
Sarmad Muhammad Soomar ${ }^{1 *}$ and Saima Rajpali ${ }^{2}$ \\ 'Senior Research Assistant, Department of Psychiatry, Aga Khan University, Karachi, Pakistan \\ ${ }^{2}$ Registered Nurse, King Faisal Specialist Hospital Riyadh, Saudi Arabia
}

Epilepsy is commonly seen in women of reproductive age and it is affecting their reproductive and pregnancy outcomes in an adverse manner [1]. It has seen that there are increased numbers of maternal deaths of pregnant women with epilepsy than those who have no any epilepsy symptoms or episodes. In many studies the common outcome observed is spontaneous miscarriage, ante partum hemorrhage or early pre term deliveries. Malformation and congenital anomalies are quite common in new born of epileptic women and also those who are on some kind of anti-epileptic medicines like valproate sodium. Death or still birth of new born babies is another complication witnessed because of epilepsy in pregnant women [2].

It is very difficult to clinically manage epileptic women. However, certain recommendations based on studies have proven very helpful if they are practiced. The major aim is this regard is balance between managing epileptic episodes and also less exposure of fetus from anti-epileptic medicines. These practice recommendations are; prophylactic use of folic acid medicine as neural tube defects can occur due to folic acid deficiency as well, checking drug levels (anti-epileptic) during pregnancy to manage at certain level, and breastfeeding babies is highly effective [3]. The direct relations are very difficult to identify in clinically diagnosed women with epilepsy. However, prior to conceiving it is highly suggested to concern their neuro-physicians.

Handling epilepsy during pregnancy is to stabilize the maternal and fetal risks related with uncontrolled seizures against the potential teratogenicity effects of antiepileptic drugs. Nowadays, researchers are moving forward in finding methods to prevent a syndrome from occurring in those who exists as high risk. Some recent basic actions include improving treatment of infections and high blood pressure during pregnancy both of which can lead to brain injury in the developing fetus [4].

During pregnancy and the intra-partum period, women are more expected to be exposed to impending seizure triggers and may have an increase in seizure incidences. Common triggers include sleep withdrawal and increased emotional stress, as well as nausea and vomiting affecting medication levels.

Pain management and the labor and delivery room environment should be optimized for the women with epilepsy. Consultation with anesthesia should be undertaken early in labor, if not prior to admission for delivery. With an appropriately dosed epidural, many women can sleep during the first stage of labor and thereby minimize the potential consequences of sleep deprivation as well as minimize painassociated stress.

Family should be encouraged to allow the laboring mother to rest and decrease external stimulation. Lighting can be lowered to encourage sleep. Risks associated with 
epilepsy during pregnancy include the potential for perinatal complications, seizure worsening, and adverse effects of antiepileptic drugs (AEDs) on the fetus and later development. Epilepsy is not a contraindication to pregnancy.

Women of child-bearing years should be counseled regarding the interactions between AEDs and hormonal contraceptive therapy, the potential risks associated with epilepsy and pregnancy, and the importance of folic acid supplementation to prevent neural tube defects. Diagnostic considerations include pregnancy-associated conditions such as eclampshia and cerebral venous thrombosis [5].

The ketogenic diet has been used for years in the treatment of epileptic conditions. Researchers are still trying to figure out how this diet works so they can simulate the treatment in other ways since this diet is so difficult to comply with. It's a high-fat, low-carbohydrate diet that is very challenging to maintain, but effective in over $50 \%$ of cases.

\section{References}

1. Galappatthy P, Liyanage CK, Lucas MN, Jayasekara DT, Abhayaratna SA, et al. Obstetric outcomes and effects on babies born to women treated for epilepsy during pregnancy in a resource limited setting: a comparative cohort study. BMC pregnancy and childbirth. 2018; 18: 230. Ref.: https://goo.gl/pcDTDM

2. Pennell PB, French JA, Harden CL, Davis A, Bagiella E, et al. Fertility and birth outcomes in women with epilepsy seeking pregnancy. JAMA Neurol. 2018; 75: 962-969. Ref.: https://goo.gl/1ZB2Ef

3. Patel SI, Pennell PB. Management of epilepsy during pregnancy: an update. Ther Adv Neurol Disord 2016; 9: 118-129. Ref.: https://goo.gl/HP6n6Z

4. Artama M, Braumann J, Raitanen J, Uotila J, Gissler M, et al. Women treated for epilepsy during pregnancy: outcomes from a nationwide population-based cohort study. Acta Obstet Gynecol Scand. 2017; 96: 812-820. Ref.: https://goo.gl/iGniSG

5. Royal College of Obstetricians and Gynaecologists (RCOG). Epilepsy in Pregnancy: Green-Top Guideline 68. 2016; 1-33. Ref.: https://goo.gl/L9xFRh 